\title{
Spectral Properties of THz-Periodic Metallic Structures
}

\author{
Chul Kang*, Chul-Sik Kee, Ik-Bu Sohn, and Jongmin Lee \\ Advanced Photonics Research Institute, Gwangju Institute Science and Technology, Gwangju, \\ 500-712, Korea
}

(Received August 19, 2008 : revised September 17, 2008 : accepted September 18, 2008)

\begin{abstract}
We have investigated spectral properties of the periodic arrays of aluminum rods and holes on papers using the terahertz time-domain spectroscopy. The size of a rod (hole) is $600 \mu \mathrm{m} \times 100$ $\mu \mathrm{m}$ and the spacing is $300 \mu \mathrm{m}$. The samples were fabricated by a femtosecond laser micromachining system. The periodic arrays of aluminum rods exhibit high reflection around $0.25 \mathrm{THz}$ when the polarization of the $\mathrm{THz}$ pulse is parallel to the long axis of the rod, whereas the periodic arrays of holes exhibit high transmission around $0.25 \mathrm{THz}$ when the polarization of the $\mathrm{THz}$ pulse is perpendicular to the long axis of the hole.
\end{abstract}

\author{
Keywords: terahertz, high reflection, Babinet's principle \\ OCIS codes : (220.4830) Optical systems design; (050.1220) Apertures; (300.6270) Spectroscopy, \\ far infrared
}

\section{INTRODUCTION}

Spectral properties of periodic metallic arrays have attracted much attention [1]. For example, the enhanced optical transmission through a metallic screen with a periodic array of holes has been observed [2-5]. The enhanced transmission has been understood by resonant coupling of surface plasmons and incident electromagnetic waves. It has also been demonstrated that periodic arrays of metallic structures could exhibit the high reflection at certain frequencies that are determined by the geometries of metallic structures [6]. The high reflection has been understood by negative effective permittivity or permeability [6-9].

Recently, properties of various materials in the terahertz $(\mathrm{THz})$ frequency range have been intensively investigated by $\mathrm{THz}$ time-domain spectroscopy (THz-TDS) [10-14]. The materials include quantum dots, carbon nanotubes, tissue, biomaterials, cancers, explosive materials, pharmacy materials, and so on. The periodic metallic structures can be good effective materials to give interesting properties in the $\mathrm{THz}$ range because the $\mathrm{THz}$ region is much less demanding than the optical region for the fabrication of sub-wavelength devices. THz transmission properties of hole-type split ring structures and

*Corresponding author: iron74@gist.ac.kr random arrays of slits in a metallic screen have been reported already [15-18]. However, periodic arrays of metallic rods in the $\mathrm{THz}$ range have rarely been investigated.

In this paper, we study the transmission properties of a periodic aluminum rod-array and a periodic hole-array in aluminum film using THz-TDS. The samples were fabricated by a femtosecond laser micromachining system. The periodic arrays of aluminum rods exhibit very low transmission around $0.25 \mathrm{THz}$ when the polarization of the $\mathrm{THz}$ pulse is parallel to the long axis of the rod, whereas the periodic arrays of holes exhibit high transmission around $0.25 \mathrm{THz}$ when the polarization of $\mathrm{THz}$ pulse is perpendicular to the long axis of the hole. The polarization-dependent transmission properties were simply explained by Babinet's principle [19].

\section{EXPERIMENT}

Figure 1 shows the periodic arrays of aluminum rods and holes which were fabricated by a commercial aluminum tape on the paper and a femtosecond laser micromachining system. The central wavelength and the width of input pulse are $785 \mathrm{~nm}$ and $184 \mathrm{fs}$, respectively, and the average power and the repetition rate are 1 watt and $1 \mathrm{KHz}$, respectively. The size of the rod (hole) is 

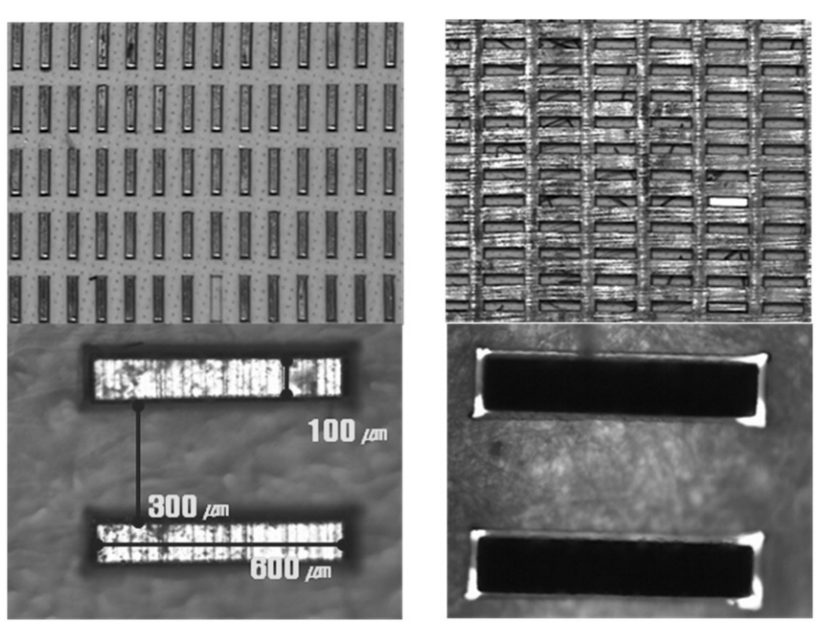

FIG. 1. Periodic aluminum rod-array and periodic holearray in aluminum film.

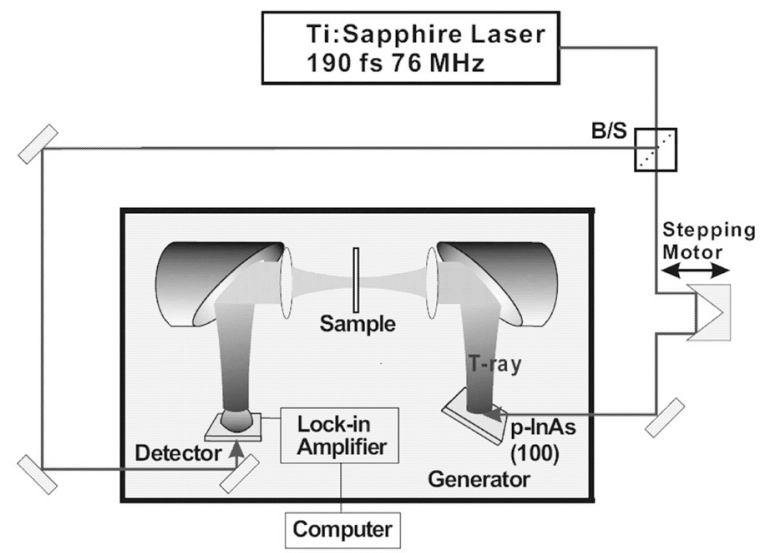

FIG. 2. Scheme of terahertz time-domain spectroscopy experimental setup.

about $600 \mu \mathrm{m} \times 100 \mu \mathrm{m}$ and the spacing between rods (holes) is about $300 \mu \mathrm{m}$. The total size of the sample is $8 \mathrm{~mm} \times 8 \mathrm{~mm}$.

The terahertz time-domain spectroscopy setup is shown Fig. 2. For the generation of $\mathrm{THz}$ pulses, the p-type (100) InAs wafer with about $10^{16} / \mathrm{cm}^{3}$ carrier concentration [20] and the femtosecond laser with the $76 \mathrm{MHz}$ repetition rate and the $190 \mathrm{fs}$ pulse width were used. The average power of the input laser is 1.3 watts and its beam diameter on the InAs wafer is $4 \mathrm{~mm}$. The 5 - $\mu \mathrm{m}$ gap dipole antenna on the low temperature grown GaAs wafer was used to detect the generated $\mathrm{THz}$ pulses.

\section{EXPERIMENTAL RESULTS}

Figure 3 shows the characteristics of THz pulses after passing through air and the paper. All measured data were acquired in the air tight box under 10-\% humidity. The signal to noise ratio is over 1000:1. In the time-
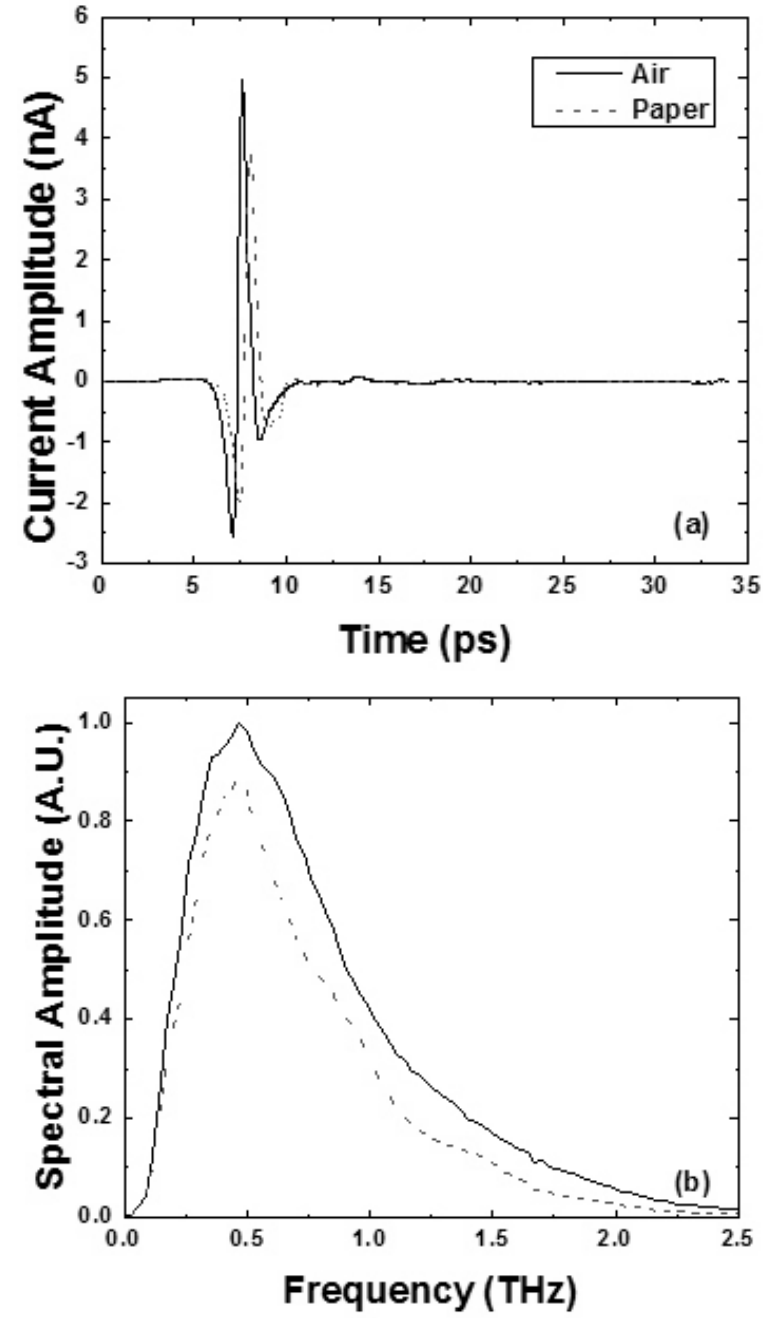

FIG. 3. Measured time-domain waveforms and their spectra in frequency domain.

domain waveforms, the peak amplitude of the $\mathrm{THz}$ pulse passed through the paper is reduced about $25 \%$ for the case of air. The phase delay between the THz pulses is about 0.5 ps. However, the spectral characteristics of $\mathrm{THz}$ pulses passed through air and the paper are almost the same.

Figure 4 (a) show the time-domain waveforms of $\mathrm{THz}$ pulses passed through the array of aluminum rods when the polarization of the $\mathrm{THz}$ pulse is parallel to the long axis of aluminum rod (red line) and perpendicular to the axis (blue line). Their spectral characteristics are shown in Fig. 4 (c). One can see that the spectrum shows very low transmission around $0.25 \mathrm{THz}$ when the polarization of the $\mathrm{THz}$ wave is parallel to the long axis of the aluminum rod. The wavelength of $0.25 \mathrm{THz}$ exactly is two times the length of a long side of rod, $(\lambda \sim 1200$ $\mu \mathrm{m})$. For the case of hole arrays, resonant transmissions are observed around $0.26(\lambda \sim 1200 \mu \mathrm{m}), 0.37(\lambda \sim 1800$ $\mu \mathrm{m})$, and $0.65 \mathrm{THz}(\lambda \sim 1800 \mu \mathrm{m})$ when the polarization of $\mathrm{THz}$ wave is perpendicular to the long axis of the 

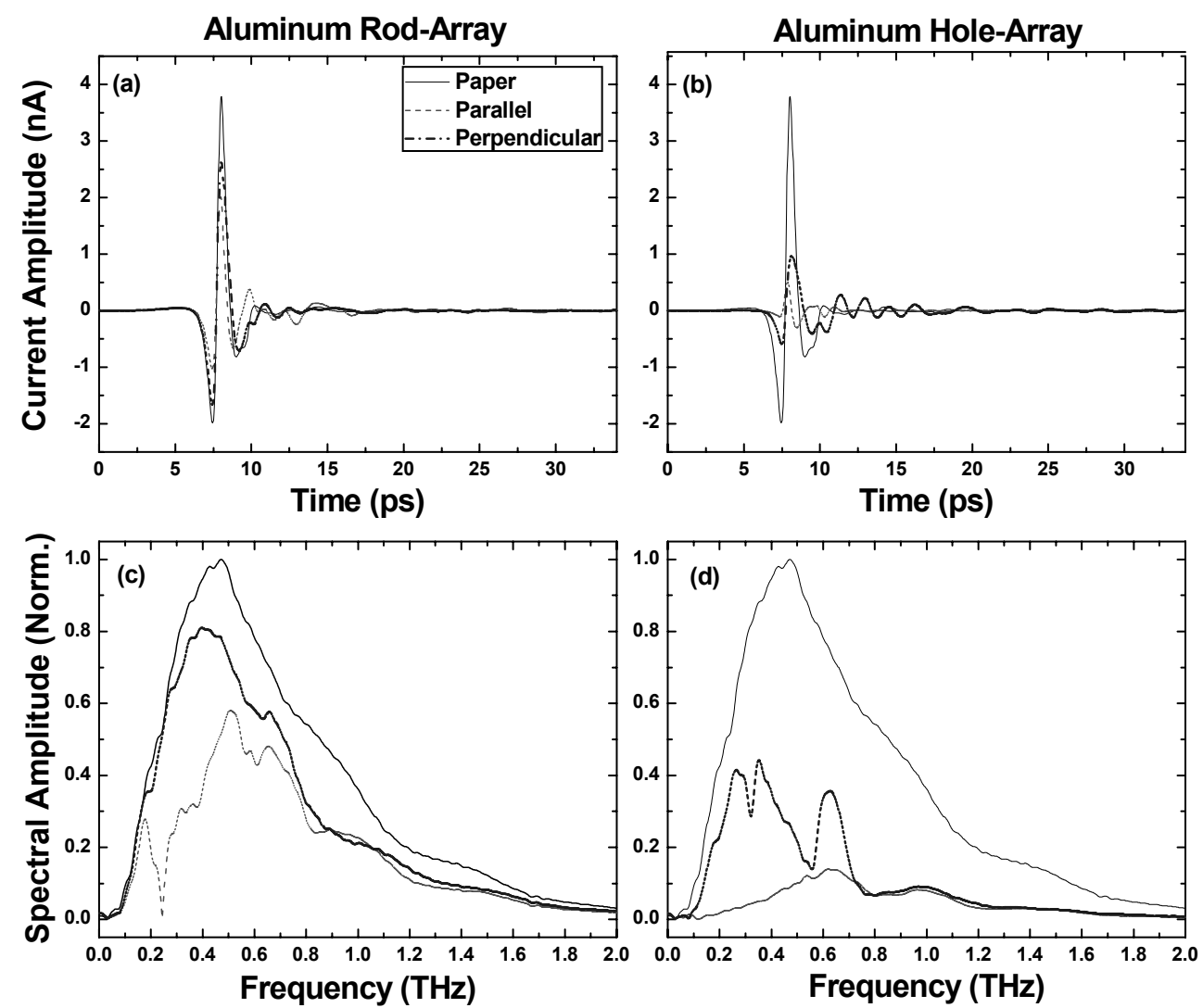

FIG. 4. Measured time-domain waveforms after passing through the aluminum rod-array and the hole-array in aluminum film (a), (b) and their spectra in frequency-domain (c), (d)

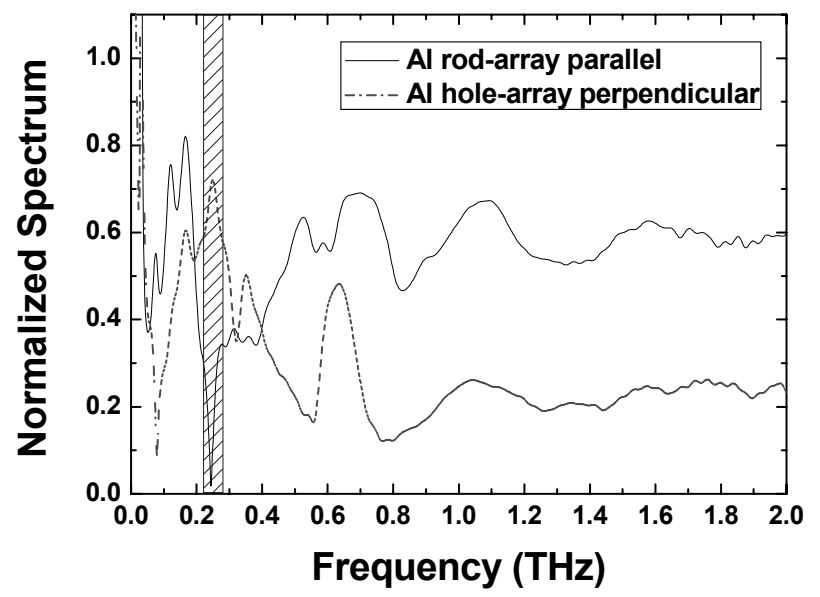

FIG. 5. Normalized transmission spectra of the rod-array for the parallel polarized $\mathrm{THz}$ pulse and of the hole-array for the perpendicularly polarized $\mathrm{THz}$ pulse.

holes.

Figure 5 shows the transmission spectra of the rodarray for the parallel polarized $\mathrm{THz}$ pulse and of the hole-array for the perpendicularly polarized $\mathrm{THz}$ pulse. The transmission intensity was normalized to that of the paper. The rod-array shows very low transmittance of $3 \%$ and the hole-array shows high transmittance of $70 \%$ at $0.25 \mathrm{THz}$. This result can be explained by Babinet's principle that has been used in understanding diffraction phenomena from an aperture in a perfect conductor screen and its complementary structure. Babinet's principle gives the relations between the transmission and reflection coefficients of the original and its complementary structures, i.e., $t_{c}=-r$ and, $r_{c}=-t$ where $t\left(t_{c}\right)$ and $r\left(r_{c}\right)$ are the reflection and the transmission coefficients of the original (complementary) structure. From the relations, one can understand qualitatively why the rod-array shows very low transmittance at $0.25 \mathrm{THz}$, while the hole-array shows high transmittance at $0.25 \mathrm{THz}$. However, we can not demonstrate quantitatively the relations because the reflection was not measured directly and there is also absorption.

\section{CONCLUSION}

Spectral properties of the periodic arrays of aluminum rods and holes have been studied by using the terahertz time-domain spectroscopy. The periodic arrays of aluminum rods exhibit very low transmission around 0.25 $\mathrm{THz}$ when the polarization of the $\mathrm{THz}$ pulse is parallel 
to the long axis of the rod, whereas the periodic arrays of holes exhibit high transmission around $0.25 \mathrm{THz}$ when the polarization of the $\mathrm{THz}$ pulse is perpendicular to the long axis of the hole.

\section{ACKNOWLEDGMENT}

This work was partially supported by Ministry of Education Science and Technology of Korea through Photonics 2020 program and Asian Laser Center of GIST.

\section{REFERENCES}

[1] F. J. Garcia de Abajo, "Colloquium: Light scattering by particle and hole arrays," Rev. Modern Phys., vol 79, no. 4, pp. 1267-1290, 2007.

[2] T. W. Ebbesen, H. J. Lezec, H. F. Ghaemi, T. Thio, and P. A. Wolff, "Extraordinary optical transmission through sub-wavelength hole arrays," Nature, vol. 301, pp. 667-669, 1997.

[3] H. F. Ghaemi, Tineke Thio, D. E. Grupp, T. W. Ebbesen and H. J. Lezec, "Surface plasmons enhance optical transmission through subwavelength holes," Phys. Rev. $B$, vol. 58, no. 11, pp. 6779-6782, 1998.

[4] L. Salomon, F. Grillot, A. V. Zayats, and F. Fronel, "Near-field distribution of optical transmission of periodic subwavelength holes in a metal film," Phys. Rev. Lett., vol. 86, no. 6, pp. 1110-1113, 2001.

[5] K. J. Klein Koerkamp, S. Enoch, F. B. Segerink, N. F. van Hulst, and L. Kuipers, "Strong Influence of Hole Shape on Extraordinary Transmission through Periodic Arrays of Subwavelength Holes", Phys. Rev. Lett., vol. 92, no. 18, pp. 183901-1 183901-4, 2004.

[6] J. B. Pendry, A. J. Holden, D. J. Robbins and W. J. Stewart, "Magnetism from conductors and enhanced nonlinear phenomena," IEEE Trans. on Microw. Theory and Tech., vol. 47, no. 11, pp. 2075-2084, 1999.

[7] J. B. Pendry, A. J. Holden, W. J. Stewart, and I. Youngs, "Extremely Low Frequency Plasmons in Metallic Mesostructures," Phys. Rev. Lett., vol. 76, no. 25, pp. 47734776, 1996.

[8] D. F. Sievenpiper, M. E. Sickmiller, and E. Yablonovitch, "3D Wire Mesh Photonic Crystals," Phys. Rev. Lett., vol. 76, no. 14, pp. 2480-2483, 1996.

[9] D. R. Smith, W. J. Padilla, D. C. Vier, S. C. Nemat-
Nasser, and S. Schultz, "Composite Medium with Simultaneously Negative Permeability and Permittivity," Phys. Rev. Lett., vol. 84, no. 18, pp. 4184-4187, 2000.

[10] K. Sakai, in the "Terahertz optoelectronics," Topics in Applied Physics, vol. 97, Springer, 2005.

[11] S. J. Oh, C. Kang, I. H. Maeng, J.-H. Son, N. K. Cho, J. D. Song, W. J. Choi, W.-J. Cho, and J. I. Lee, "Measurement of carrier concentration captured by InAs/GaAs quantum dots using terahertz time-domain spectroscopy," Appl. Phys. Lett., vol. 90, no. 13, pp. 131906-1 1319063, 2007.

[12] Kang, I. H. Maeng, S. J. Oh, J. H. Son, T. I. Jeon, K. H. An, S. C. Lim, and Y. H. Lee, "Frequency-dependent optical constants and conductivities of hydrogenfunctionalized single-walled carbon nanotubes," Appl. Phys. Lett., vol. 87, no. 4, pp. 041908-1 041908-3, 2005.

[13] E. Pickwell and V. P. Wallace, "Biomedical applications of terahertz technology," J. Phys. D: Appl. Phys., vol. 39, no. 17, pp. R301-R310, 2006.

[14] C. Baker, T. Lo, W. R. Tribe, B. E. Cole, M. R. Hogbin, and M. C. Kemp, "Detection of Concealed Explosives at a Distance Using Terahertz Technology," Proc. IEEE, vol. 95, no. 8, pp. 1559-1565, 2007.

[15] J. Han, X. Lu, and W. Zhang, "Terahertz transmission in subwavelength holes of asymmetric metal-dielectric interfaces:The effect of a dielectric layer," J. Appl. Phys., vol. 103, no. 13, pp. 033108-1 033108-4, 2008.

[16] J. W. Lee, M. A. Seo, D. H. Kang, K. S. Khim, S. C. Jeoung and D. S. Kim, "Terahertz Electromagnetic Wave Transmission through Random Arrays of Single Rectangular Holes and Slits in Thin Metallic Sheets," Phys. Rev. Lett., vol. 99, no. 13, pp. 137401-1 1374014, 2007.

[17] J. W. Lee, M. A. Seo, D. J. Park, S. C. Jeoung, Ch. Lienau, Q-Han Park, P. C. M. Planken, and D. S. Kim, "Shape Resonance Omni-directional Terahertz Filters with Near-unity Transmittance," Opt. Exp., vol. 14, no. 3, pp. 1253-1259, 2006

[18] A. J. L. Adam, J. M. Brok, M. A. Seo, K. J. Ahn, D. S. Kim, J. H. Kang, Q. H. Park, M. Nagel, and P. C. M. Planken, "Advanced terahertz electric near-field measurements at sub-wavelength diameter metallic apertures," Opt. Exp, vol. 16, no.10, pp.7407-7417, 2008.

[19] M. Born and E. Wolf, "Principles of optics: Electromagnetic theory of propagation, interference and diffraction of light," (Cambridge University Press, Cambridge, England, 1999.)

[20] K. Liu, J. Xu, T. Yuan, and X.-C. Zhang, "THz radiation from InAs induced by carrier diffusion and drift," Phys. Rev. B, vol. 73, pp. 155330-155334, 2006. 